\title{
EVOLUTION OF DISTRIBUTION FUNCTION FOR COSMOLOGICAL NEUTRINO
}

\author{
HYUNG WON LEE \\ Institute of Basic Science and School of Computer Aided Science \\ Inje University, Inje-ro 197, Gimhae 621-749, Korea \\ hwlee@inje.ac.kr \\ KYOUNG YEE KIM \\ Institute of Basic Science and School of Computer Aided Science \\ Inje University, Inje-ro 197, Gimhae 621-749, Korea \\ kimky@inje.ac.kr \\ REMO RUFFINI \\ ICRANet, Piazzale della Repubblica, 10-65100 Pescara, Italy \\ ruffini@icra.it
}

\begin{abstract}
Recent experimental evidences show that the universe is accelerating. There are many modified cosmological models to explain this accelerating phase including $\Lambda \mathrm{CDM}$, quintessence model, Brans-Dicke, $f(R)$ gravity, and others. In this talk we want to check the possibility of explaining the current accelerating phase using standard cosmological model with degenerate neutrino. We will study the evolution of distribution function of degenerate neutrinos with standard Friedmann-Lemaître-Roberston-Walker(FLRW) background.
\end{abstract}

Keywords: Neutrino; degenerate neutrino; cosmological evolution.

PACS numbers: 04.20.-q, 04.20.Jb

\section{Introduction}

Especially, the degenerate neutrinos have unique effect for the evolution of universe with its interactions with other fundamental particles and affecting expansion rates. ${ }^{1}$ Recently, it has been preferred that the composition of the Universe is $25 \%$ of cold dark matter, $75 \%$ of dark energy and the very small part of radiation matter. Furthermore, the universe is in the accelerating phase currently. ${ }^{2,3}$ This ansatz will be critically exercised. There are many cosmological models to explain this accelerating Universe but fundamental explanation. ${ }^{4}$ In this series of articles we want to try to explain this phenomenon with degenerate neutrino. Firstly, neutrino was thought as a hot dark matter candidate; however, with its ultra relativistic properties it could not explain the current small scale structures. But most of the analysis is assumed for non-degenerate massless neutrinos. We know that neutrinos have non 
zero mass by various experiments. ${ }^{5}$ Also for degenerate neutrinos, their behaviour will be different and affect the evolution of the universe differently. As the first article for the series, here we will confine ourselves to study only for basic properties of degenerate neutrinos and their possible effect for cosmological perturbation evolution.

\section{Accelerating Cosmological Evolution}

Imposing homogeneous and isotropic condition for universe, we can use the standard Friedmann-Lemaître-Roberston-Walker(FLRW) metric for cosmology.

$$
d s^{2}=-c^{2} d t^{2}+a(t)^{2}\left(d r^{2}+r^{2} d \Omega^{2}\right) .
$$

Using standard Einstein-Hilbert action,

$$
S=\frac{1}{8 \pi G} \int d^{4} x \sqrt{-g} R+I_{\mathrm{m}},
$$

we obtain the Hubble equation

$$
H^{2}=\frac{8 \pi G}{3 c^{2}} \rho
$$

where $\rho$ is the total energy density of the cosmological fluid. Total energy density could be written as the sum of each component of the cosmological fluid,

$$
\rho=\rho_{\mathrm{CDM}}+\rho_{\mathrm{rad}}+\rho_{\nu \bar{\nu}},
$$

where $\rho_{\mathrm{CDM}}, \rho_{\mathrm{rad}}$, and $\rho_{\nu \bar{\nu}}$ are constributions of standard cold dark matter with equation of state is zero, radiation matter with $1 / 3$, and degenerate neutrino respectively. Note that here we do not introduce any exotic matter or modification of standard Einstein relativity to explain cosmological acceleration.

One can write Eq. (3) as follows emphasizing the contributions of each components,

$$
H^{2}=H_{\mathrm{CDM}}^{2}+H_{\mathrm{rad}}^{2}+H_{\nu \bar{\nu}}^{2} .
$$

On the other hand, the conservation law for each component of fluid could be written as

$$
\dot{\rho}_{\mathrm{X}}+3 H\left(1+w_{\mathrm{X}}^{\mathrm{eff}}\right) \rho_{\mathrm{X}}=0,
$$

where $w_{\mathrm{X}}^{\text {eff }}$ is the effective equation of state for fluid component $\mathrm{X}$ and the subscript $\mathrm{X}$ is one of CDM, rad, and $\nu \bar{\nu}$. This equation could be integrated easily to give a solution

$$
\rho_{\mathrm{X}}=\rho_{\mathrm{X}}^{0} e^{-3 \int_{0}^{x}\left(1+w_{\mathrm{X}}^{\mathrm{eff}}\left(x^{\prime}\right)\right) d x^{\prime}},
$$

where $x=\ln \left(\frac{a}{a_{0}}\right)$, with $a_{0}$ is the current scale factor and $\rho_{\mathrm{X}}^{0}$ is the current energy density of $\mathrm{X}$ component. This equation could be written as

$$
\rho_{\mathrm{X}}=\rho_{\mathrm{X}}^{0}\left(\frac{a}{a_{0}}\right)^{-3\left(1+w_{\mathrm{X}}^{\mathrm{eff}}\right)},
$$


if $w_{\mathrm{X}}^{\mathrm{eff}}$ is independent to $x$ as CDM and radiation matter cases. Understanding the contribution of each component to Hubble parameter as

$$
H_{\mathrm{X}}^{2}=\frac{8 \pi G}{3 c^{2}} \rho_{\mathrm{X}}
$$

one can easily find the corresponding equation of state as

$$
w_{\mathrm{X}}^{\mathrm{eff}}=-1-\frac{1}{3} \frac{d \ln \rho_{\mathrm{X}}}{d x}=-1-\frac{1}{3} \frac{d \ln H_{\mathrm{X}}^{2}}{d x} .
$$

For the case of $H_{\mathrm{CDM}}^{2}=\frac{8 \pi G}{2 c^{2}} H_{0}^{2} \Omega_{\mathrm{CDM}}^{0} e^{-3 x}$, one can get $w_{\mathrm{CDM}}^{\mathrm{eff}}=0$ and for $H_{\mathrm{rad}}^{2}=$ $\frac{8 \pi G}{2 c^{2}} H_{0}^{2} \Omega_{\mathrm{rad}}^{0} e^{-4 x}, w_{\mathrm{CDM}}^{\mathrm{eff}}=1 / 3$ as expected.

If some component of cosmological fluid contributes to Hubble parameter as

$$
H_{\mathrm{X}}^{2}=\frac{8 \pi G}{3 c^{2}} H_{0}^{2}\left(f_{0}+f_{1} e^{\alpha x}\right)
$$

where $f_{0}, f_{1}$, and $\alpha$ are some dimensionless constants. It cosrespond to its density is given by

$$
\rho_{\mathrm{X}}=H_{0}^{2}\left(f_{0}+f_{1} e^{\alpha x}\right),
$$

Then its equation of state is given as

$$
w_{\mathrm{X}}^{\mathrm{eff}}=-1-\frac{\alpha}{3} \frac{f_{1} e^{\alpha x}}{f_{0}+f_{1} e^{\alpha x}},
$$

Note that the parameter $f_{1}$ should satisfy the relation

$$
f_{1}=1-\Omega_{\mathrm{m}}^{0}-\Omega_{\mathrm{r}}^{0}-f_{0},
$$

where $\Omega_{\mathrm{m}}^{0}$ is the current value of density parameter for cold dark matter and $\Omega_{\mathrm{r}}^{0}=$ $\chi \Omega_{\mathrm{m}}^{0}$ for radiation matter, which is determined by observation, to satisfy the first Hubble equation $\Omega_{\mathrm{m}}+\Omega_{\mathrm{r}}+\Omega_{\mathrm{X}}=1$. The behaviours of density parameters with cosmological evolution are given in Fig. 2 for three cases.

If we define the effective equation of state for the universe as $w^{\text {eff }}$ satisfying the equation

$$
\dot{\rho}+3 H\left(1+w^{\mathrm{eff}}\right) \rho=0,
$$

where $r h o$ is the total energy density of the universe. The $w^{\text {eff }}$ can be obtained as

$$
w^{\mathrm{eff}}=-1-\frac{1}{3} \frac{\ln \rho}{d x}
$$

which can be rewritten as

$$
w^{\mathrm{eff}}=-1-\frac{\alpha}{3} \frac{f_{1} e^{\alpha x}}{f_{0}+f_{1} e^{\alpha x}}+\Omega_{\mathrm{m}}+\frac{4}{3} \Omega_{\mathrm{r}}
$$

The behaviours of $w_{\mathrm{X}}^{\mathrm{eff}}$ and $w^{\mathrm{eff}}$ with cosmological evolution are given in Fig. 2 for three cases as in Fig. 2.

From Figs. 1 and 2, one can understand that any cosmological fluid more slowly decaying than dust matter could be dominated after some epoch. However, the cosmological acceleration, $w^{\text {eff }}<-\frac{2}{3}$, only possible with some constant term $\left(f_{0} \neq\right.$ $0)$ or $\alpha>-1$. 


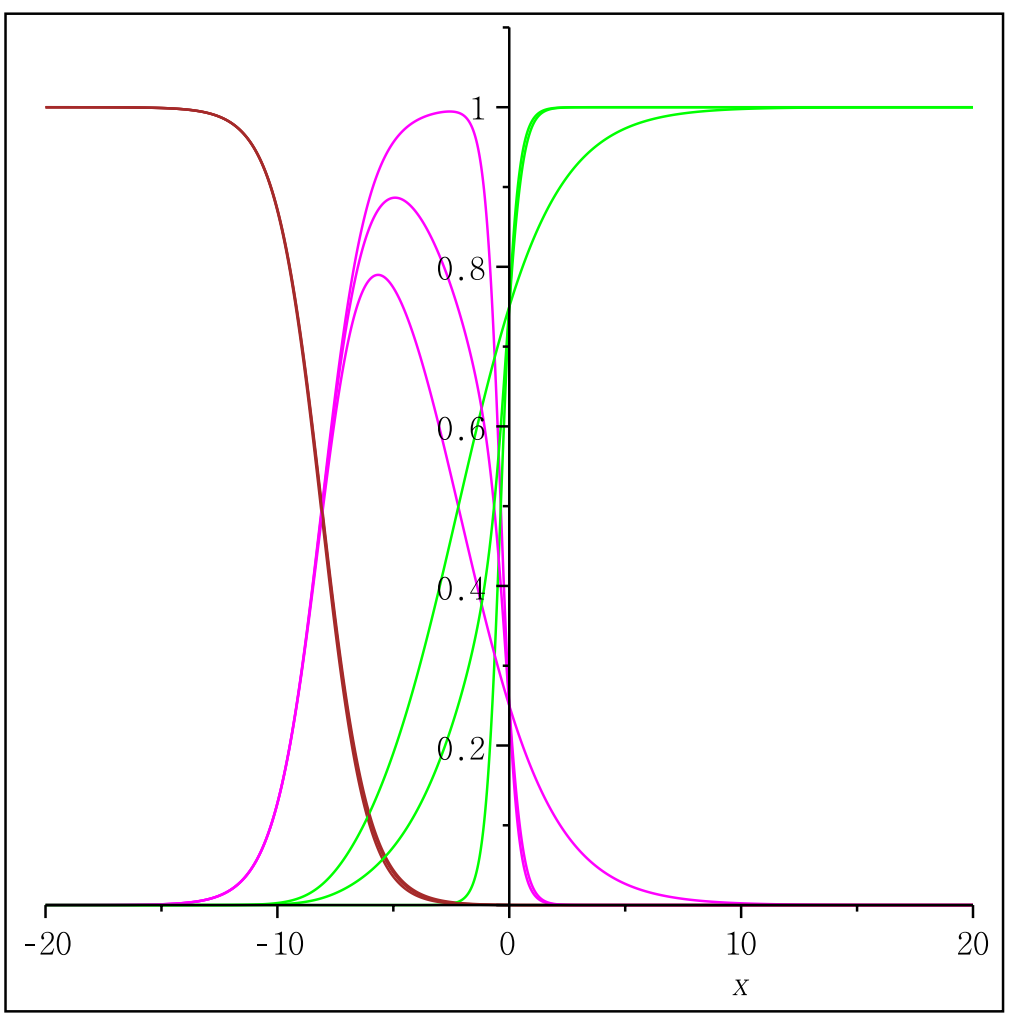

Fig. 1. A typical evolutions for density parameters, cold dark matter(magenta), radiation matter(brown), and dark energy(green). We have shown for the parameters as $\Omega_{\mathrm{m}}^{0}=0.25, \chi=$ $3.1 \times 10^{-4}$, and $\left(\alpha=0, f_{0}=0.75\right)$ corresponding to $\Lambda$ CDM (uppermost case for magenta in the graph $),\left(\alpha=-2.5, f_{0}=0.5\right)$ corresponding to $\Lambda$ (cosmological constant) + slowly decaying matter(middle case for magenta in the graph), $\left(\alpha=-2.5, f_{0}=0.0\right)$ corresponding to slowly decaying matter(lowest case for magenta in the graph).

\section{Cosmological Implications for Degenerate Massive Neutrinos}

In the previous section, it has been shown that any cosmological fluid more slowly decaying than dust matter could behave like the dark energy and give current accelerating expansion. Here we want to describe the possibility the degenerate massive neutrino to be a such kind of cosmological fluid. The main concerning is that we want to find a plausible explanation of accelerating universe without introducing any exotic matter and/or modification of the standard Einstein relativity.

The neutrinos were considered as massless particle for long time, but recent neutrino oscillation experiment proved indirectly it is massive. ${ }^{6}$ Since they interact very weakly with ordinary matter, it was also considered as a natural candidate for dark matter. It was shown that the neutrino is not viable as a dark matter, since it could not explain small scale strutures and could not close the universe energy 


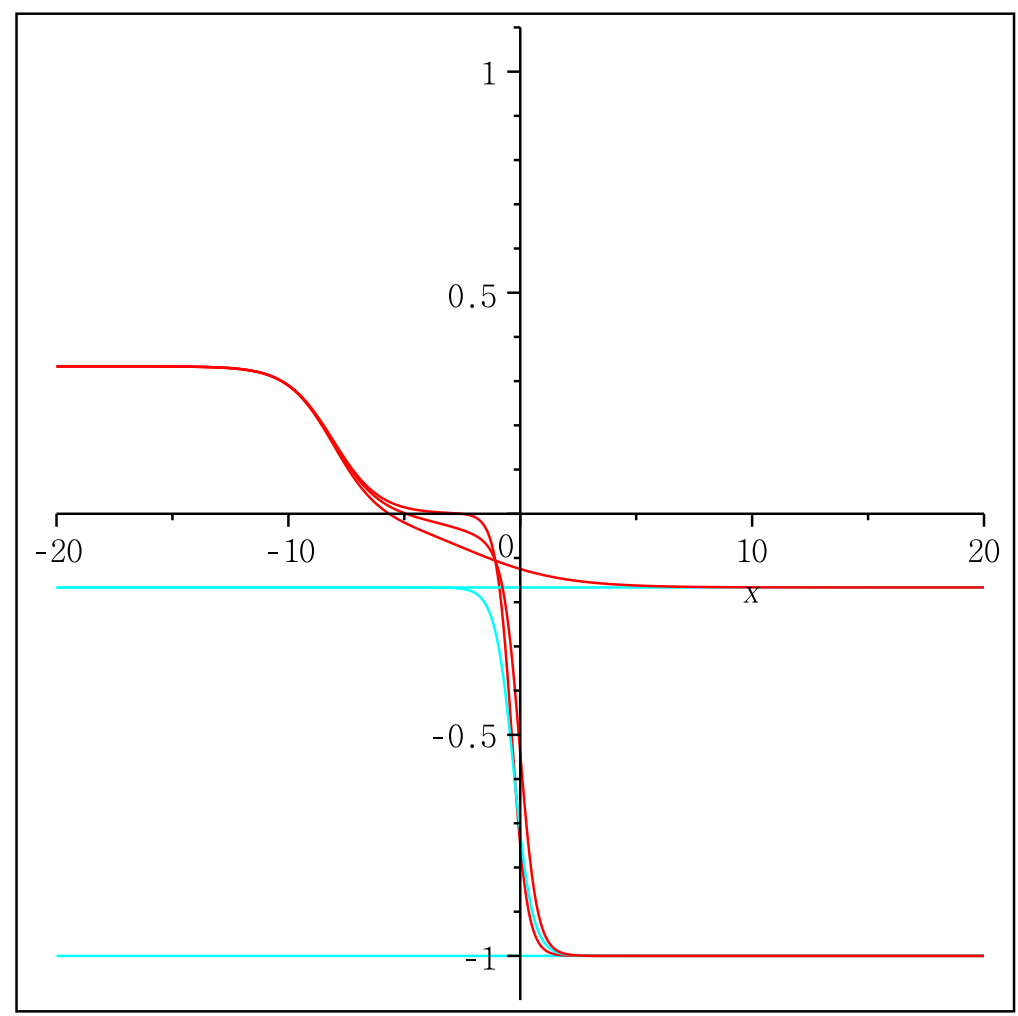

Fig. 2. A typical evolutions for the effective equations of states, for dark energy(cyan) and for total energy(red). We have shown for the parameters as $\Omega_{\mathrm{m}}^{0}=0.25, \chi=3.1 \times 10^{-4}$, and $(\alpha=0$, $\left.f_{0}=0.75\right)$ corresponding to $\Lambda \mathrm{CDM}$ (lowest constant cyan line), $\left(\alpha=-2.5, f_{0}=0.5\right)$ corresponding to $\Lambda$ (cosmological constant) + slowly decaying matter(varying cyan line in the graph, varying from $-\frac{1}{6}$ to -1$),\left(\alpha=-2.5, f_{0}=0.0\right)$ corresponding to slowly decaying matter(upper constant cyan line in the graph, its value is $\left.-1-\frac{-2.5}{3}=-\frac{1}{6}\right)$.

density. ${ }^{7}$ However, if we allow the degeneracy of the neutrinos, it could close the energy density of the universe. ${ }^{1}$

Using the red-shift distribution function, the energy density of the degenerate massive neutrio is given by

$$
\begin{aligned}
\rho_{\nu, \bar{\nu}}(t) & =\int \frac{d^{3} p}{(2 \pi)^{3}} \sqrt{p^{2}+m^{2}} f_{\nu, \bar{\nu}}(p, t), \\
f_{\nu, \bar{\nu}}(p, t) & =\frac{1}{\exp \left(\frac{\sqrt{a^{2}(t) p^{2} / a_{D}^{2}+m^{2}}}{T} \pm \xi\right)+1},
\end{aligned}
$$

where $a_{D}$ is the scale factor at neutrino decoupling, $\xi=\mu / T$ is degeneracy parameter. Unfortunately, the time dependence of neutrino density given by Eq. 18 does not a satisfacory result as shown in Fig. 3. That is, neutrino become dominat in both very early and late time evolution. 


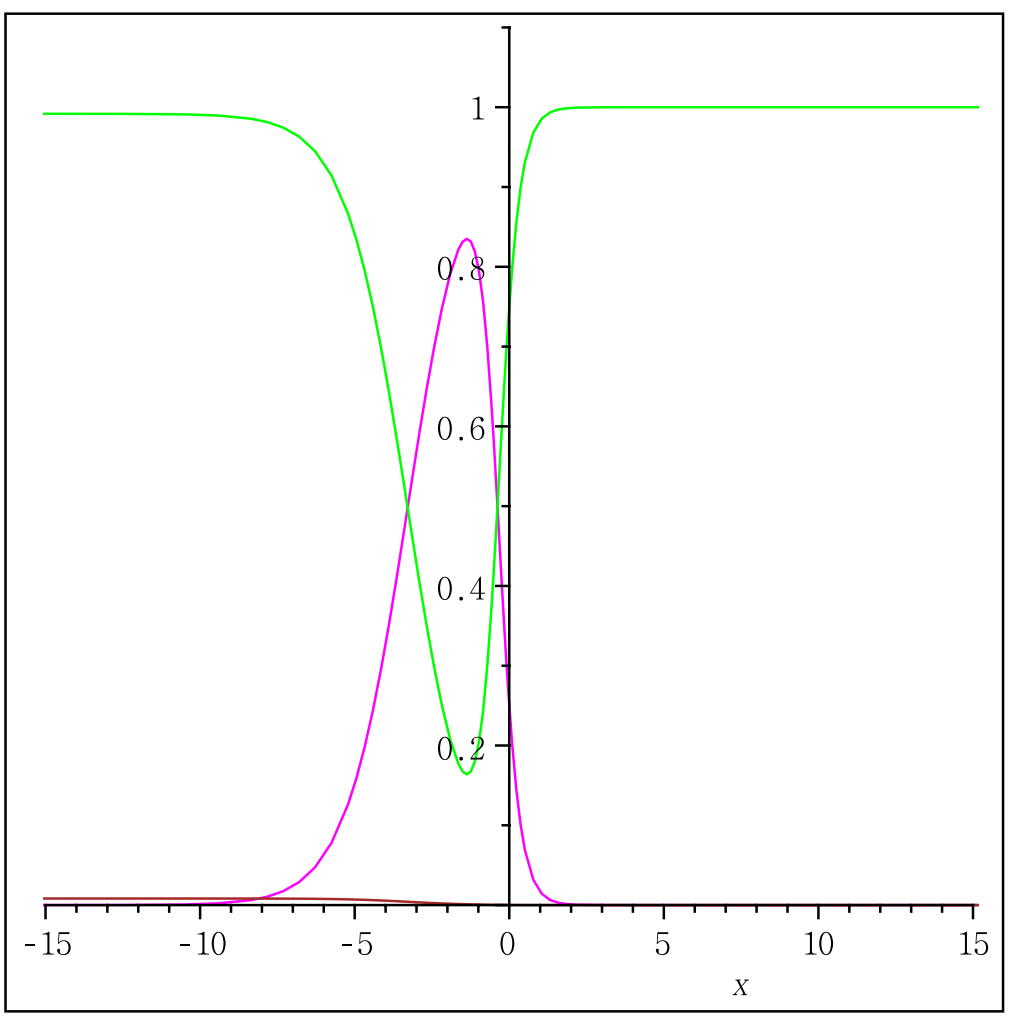

Fig. 3. A typical evolutions for density parameters, cold dark matter(magenta), radiation matter(brown), and neutrino(green). We have shown for the parameters as $\Omega_{\mathrm{m}}^{0}=0.25, \chi=3.1 \times 10^{-4}$, and $\xi=1$. Note that neutrino dominate in both at the very early and late time evolution.

This is the very preliminary result. It should be performed more detailed analysis required to discard degenerate massive neutrino as a viable dark enenrgy candidate including correct Boltzmann equation treatment, ferminonic property of the neutrino, and temperature effect on massive neutrinos. We expect that quantum mechanical zero temperature energy behave as a cosmological constant and the fermionic property could give slowly decaying energy density. A detailed and rigorous study for this points will be given elsewhere.

\section{Discussions}

We have test the possibility of degenerate massive neutrino to be dark energy comoponent. It has been shown that a simpe red-shift distribution function for neutrino is not sufficient to give viable property. However, this assumption is too simple to get correct description for accelerating universe. We need more detail analysis for the evolution of distribution function and quantum mechanical property of degenerate massive neutrino at low temperature(late evolution time). Such kind of detail study will be given in future works. 


\section{Acknowledgments}

H.W. Lee was supported by the International Research \& Development Program of the National Research Foundation of Korea(NRF) funded by the Ministry of Education, Science and Technology(MEST) of Korea(Grant number: K2011.00007, FY2011).

\section{References}

1. A. Bianconi, H. W. Lee, and R. Ruffini, Atron. Astrophys. 241, 343 (1991).

2. S. Perlmutter, M. S. Turner, and M. J. White, Phys. Rev. Lett. 83, 670 (1999), astro-ph/9901052.

3. A. G. Riess et al. (Supernova Search Team), Astrophys. J. 607, 665 (2004), astro-ph/0402512.

4. T. P. Sotiriou and V. Faranoi, Rev. Mod. Phys. 82, 452 (2010).

5. E. W. Otten and C. Weinheimer, Rept. Prog. Phys. 71, 086201 (2008), 0909.2104.

6. T. Kajita (Kamiokande) (1992), prepared for International Symposium on Neutrino Astrophysics, Takayama / Kamioka, Japan, 19-22 Oct 1992.

7. O. Elgaroy and O. Lahav, New J. Phys. 7, 61 (2005), hep-ph/0412075. 